\title{
Variations of flow in human airways as a consequence of lung diseases
}

\author{
Frantisek Lizal ${ }^{1, *}$, David Stejskal ${ }^{1}$, Miloslav Belka ${ }^{1}$, Jan Jedelsky ${ }^{1}$, Miroslav Jicha ${ }^{1}$, Kristian Brat $^{2}$, Vladimir Herout $^{2}$ and \\ Elena Lizalova Sujanska ${ }^{2}$ \\ ${ }^{1}$ Brno University of Technology, Faculty of Mechanical Engineering, Energy Institute, Technicka 2896/2, Brno 616 69, Czech Republic \\ ${ }^{2}$ The University Hospital Brno, Clinic of Pulmonary Diseases and Tuberculosis, Jihlavska 20, Brno 625 00, Czech Republic
}

\begin{abstract}
The efficiency of drug delivery administered by inhalation depends, among other factors, such as size and shape of aerosol particles, significantly also on the flow in the airways. As many lung diseases change both the breathing pattern and the shape of airways, we focus in this study on the influence of several selected diseases on the distribution of flow between the lung lobes and on changes the diseases induce on the course of flowrate. First, we present results of a literature survey focused on the published records of pathological breathing patterns. In the second part, we describe the newly designed breathing simulator and the implementation of the patterns into it. The last part is focused on the experimental verification of fidelity of the simulated breathing patterns.
\end{abstract}

\section{Introduction}

A proper delivery of a prescribed medication to afflicted lung regions is a crucial point of the therapy of respiratory diseases. The efficiency of the drug delivery administered by inhalation depends, among other factors, such as size and shape of aerosol particles, also on the flow in the airways. However, our ability to predict the distribution of inhaled particles in diseased airways is insufficient [1].

Human airways consist of the oral (and nasal) cavity, pharynx, larynx, trachea and 23 generations of bronchial branching. The lungs are divided into five lobes, while two lobes are formed in the left lung and three lobes are formed in the right lung. The respiratory cycle comprises the active action of inspiration, which is driven by the diaphragm and intercostal muscles, and the passive phase of expiration, which uses natural elasticity of the lungs and thorax.

\subsection{Lung volumes and capacities}

The lung function monitoring and diagnostics of certain diseases can be performed using lung volumes and lung capacities. The volume of air that is inhaled and exhaled during one breath cycle under resting conditions is called tidal volume $(T V)$. Its value is around 0.51 for healthy adults. Residual volume $(R V)$ is the volume of air remaining in the lungs after a maximal exhalation. Inspiratory reserve volume $(I R V)$ is the maximal volume that can be inhaled from the end-inspiratory level. Inspiratory capacity $(I C)$ is the volume of air that can be inhaled after normal exhalation. It is the sum of $I R V$ and
$T V$. Expiratory reserve volume $(E R V)$ is the maximal volume of air that can be exhaled from the normal endexpiratory position. Vital capacity $(V C)$ is the volume of air which can be exhaled after the deepest inhalation. It is the sum of $I R V, E R V$ and $T V$. Total lung capacity $(T L C)$ is the volume in the lungs at maximal inflation, the sum of $V C$ and $R V$.

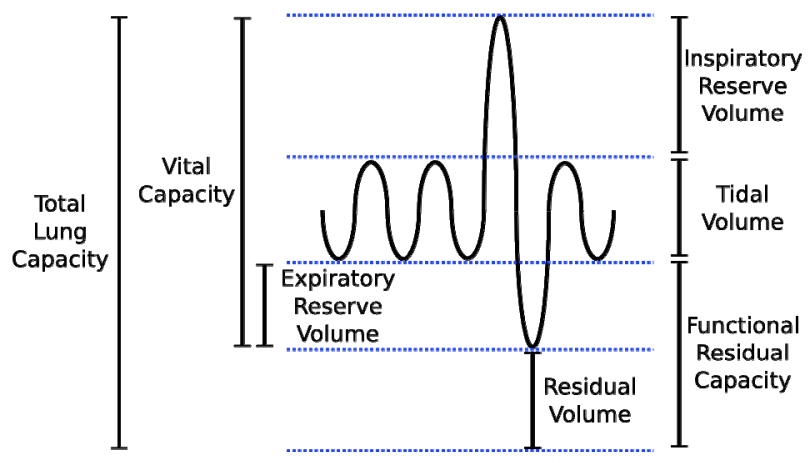

Fig. 1. An illustration of the lung volumes and capacities [2].

Functional residual capacity (FRC) is the volume in the lungs at the end-expiratory position. Forced vital capacity (FVC) is the maximal volume of air that can be exhaled after a deep inspiration. Unlike the VC, which is measured during the quiet breathing, the $\mathrm{FVC}$ is measured during vigorous forced expiration. FVC is a dynamic value. Forced expiratory volume (FEV1) is the volume of exhaled air during the first second of forced expiration. FEV1 is a dynamic value and is usually given as a percentage of FVC. 


\subsection{Measurement of the lung volumes and capacities}

The data on the flow and inhaled air volume distribution among the lobes presented in this text originate from several measuring methods. The most common method is spirometry (see Fig. 2), which serves as a main pulmonary function test in the clinical practice.

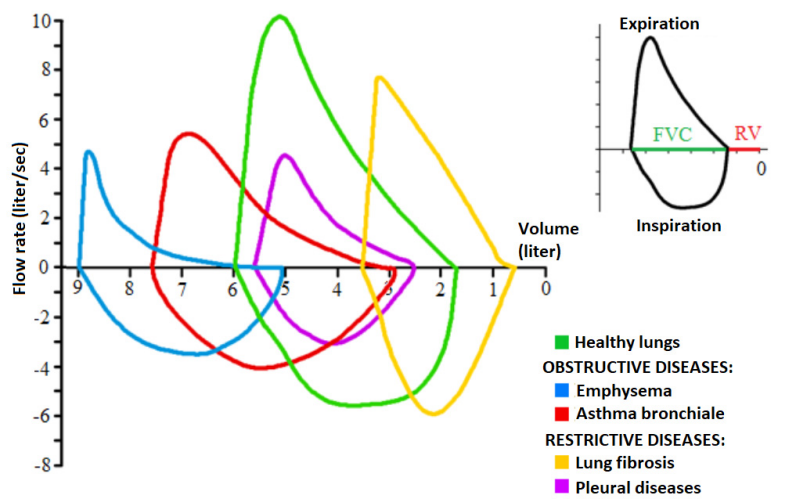

Fig. 2. Flow-Volume loop (output of the spirometry) showing FVC manoeuvre for healthy and diseased lungs [3].

All the major lung volumes and capacities except RV and FRC can be measured using spirometry, however, only the data for whole lungs are obtained, and hence the volume distribution among the lobes must be acquired using different methods.

Such a suitable method is Computed Tomography (CT), which can be performed even for dynamic characteristics, in the case that the CT scanner allows for fast volumetric imaging. As supplementary methods also Positron Emission Tomography (PET) and Single Photon Emission Computed Tomography (SPECT) can be used [4].

\subsection{Respiratory diseases and their influence on the lung functions}

Major inter-subject variability in particle deposition in the airways (up to 30\%) exists among healthy individuals [5]. This variability is highlighted during pathological conditions represented by different respiratory diseases including bronchial asthma, chronic obstructive pulmonary disease (COPD), interstitial lung diseases (ILDs) or cystic fibrosis (CF) [5]. Both bronchial asthma and COPD patients suffer with bronchial obstruction, i.e. limitation of airflow in the lower airways. Differences in breathing cycles of healthy peers and asthmatic patients were studied by Choi et al. [6]. The pathological changes of airflow and lung mechanics in COPD and asthma patients increase rapidly during periods of disease progression (i.e. exacerbation of asthma/COPD). Moreover, in many COPD patients, multiple areas of emphysema develop during the course of the disease, causing dramatic changes in the lung architecture. Abnormal patterns of particle deposition are an obvious consequence of this process in patients with this group of pulmonary diseases.
In contrast, the group of ILDs and CF are diseases characterized by a progressive accumulation of collagen fibres in the pulmonary intercellular space. This process results in dynamic loss of lung elasticity and compliance as well as in the development of bronchiectases, i.e. aneurysmatic remodelling of the bronchial lumina. In consequence, the patterns of particle deposition may change very dramatically in course of the disease. Importantly, inhalation of hypothetic new agents might be one of the potential ways to target mechanisms of the fibrotic process effectively. Thus, more precise data are warranted to better understand the progressive and longterm impacts of ILDs and CF on lung mechanics and bronchial airflow.

For study purposes (focusing on ILDs and obstructive pulmonary diseases), computed tomography (CT scanning) and magnetic resonance imaging (MRI) of the lungs are most widely used to follow-up the patterns of pathological changes in bronchial or lung architecture [7]. For illustration, CT scans of normal lungs and of lungs of a patient with $\mathrm{CF}$ are presented as Figs. 3 and 4, respectively. However, obtaining data on ILDs and CF lobar flow distribution is difficult, therefore we decided to begin with diseases for which the data were already published.

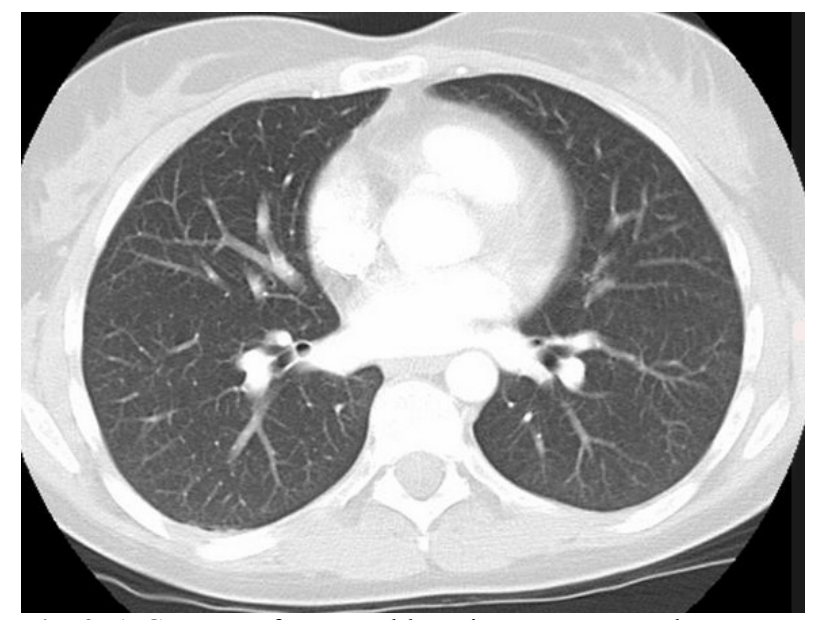

Fig. 3. A CT scan of a normal lung in a transverse plane.

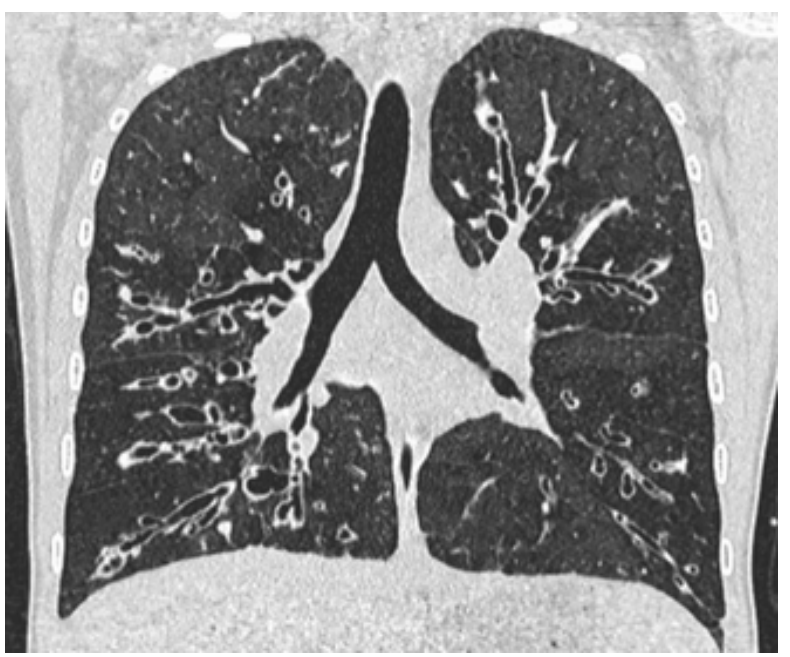

Fig. 4. A CT scan of a patient with multiple bronchiectasis in a coronal plane (clinical diagnosis: cystic fibrosis). 
The main aim of this work is to acquire data on flow and inhaled air volume distribution among the lung lobes and implement these into a newly designed breathing simulator. The simulator will be employed during experiments studying regional deposition of aerosols in human airway replicas [8]. As the differences among the healthy and diseased lungs are less apparent during normal breathing, and also due to the fact, that inhalation of medical aerosols follows a pattern significantly different than normal breathing, we decided to set up and simulate the IC manoeuvre. After implementation of the obtained breathing patterns into the simulator it was necessary to verify the ability of the simulator to follow the selected regime and to close the electro-pneumatic valves to allow separation of inspiration and expiration phase for aerosol and clean air path.

\section{Methods}

The whole process of obtaining, processing and implementation of the breathing patterns was described in the bachelor thesis of David Stejskal [3]. Briefly, as a first step the distribution of volumes among the lobes for healthy lungs during normal breathing and $I C$ manoeuvre were obtained from the literature $[6,9]$. Then the data for several diseases were acquired. The data for asthmatics were obtained also from the study of Choi et al. [6], the data on emphysema were from [10], on cancer from [11] and on lobectomy from [12].

\subsection{Breathing simulator setup}

The newly designed breathing simulator (custom-built, based on FESTO technology, Esslingen am Neckar, Germany) comprises five computer-controlled motors which drive pistons in five pairs of cylinders. One cylinder is always for the clean air, the second serves for the aerosol path. This division is necessary, as separate measurement of inspiration and expiration (and hence separate dosing of aerosol into inspiration and expiration phase) is required.

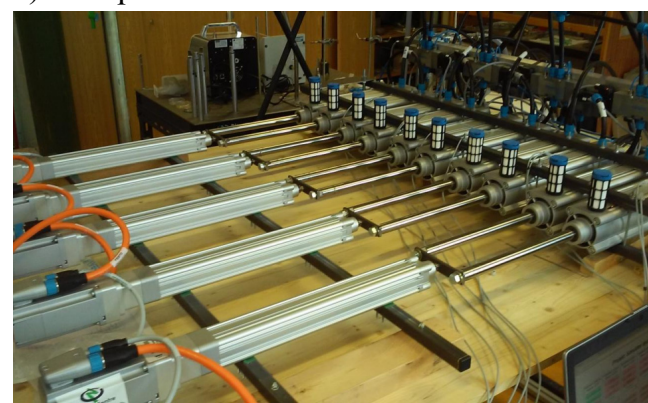

Fig. 5. A photograph of the breathing simulator - motors and pneumatic cylinders.

The obtained patterns were normalized in their duration and distribution of flows and volumes among the lobes were prescribed. The resulting courses of flowrates were transformed into prescription of piston positions for each lobe (see examples in Figures 6 to 8, where LUL means left upper lobe, LLL - left lower lobe, RUL - right upper lobe, RML - right middle lobe and RLL - right lower lobe).

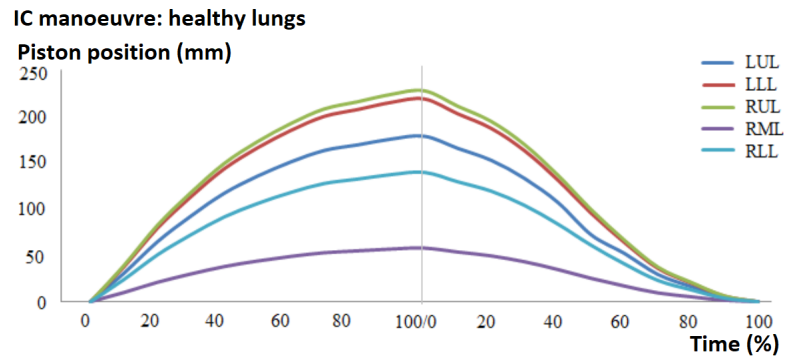

Fig. 6. $I C$ manoeuvre - transformation of the flow distribution among the lobes to the prescription of the piston position for healthy lungs.

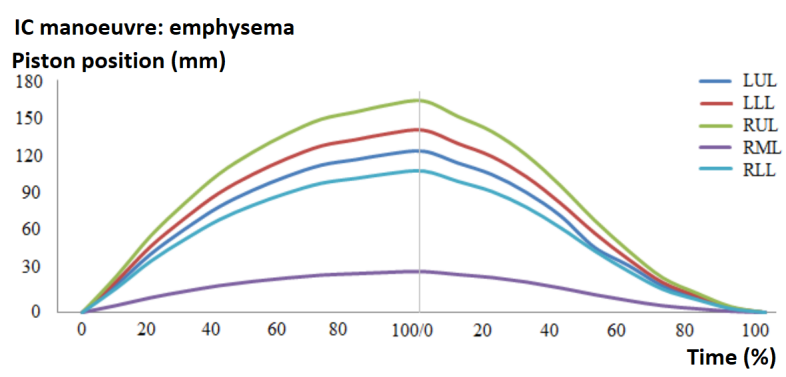

Fig. 7. IC manoeuvre - transformation of the flow distribution among the lobes to the prescription of the piston position for lungs with emphysema.

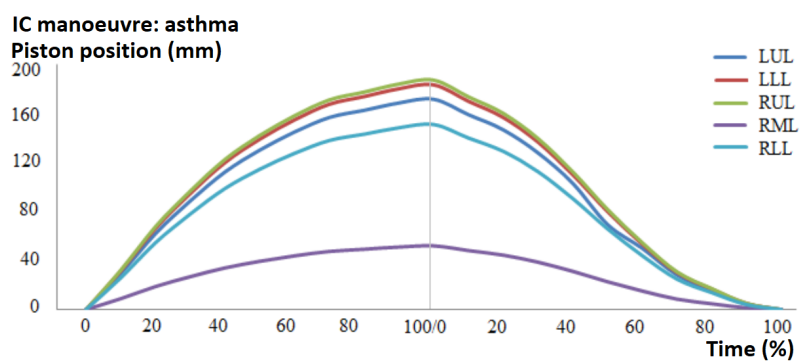

Fig. 8. IC manoeuvre - transformation of the flow distribution among the lobes to the prescription of the piston position for severe asthma.

\subsection{Measurement setup}

The duration of switching of the closing valves on the pneumatic cylinders, which is important for accurate separation of the inspiratory and expiratory phase during experiments with aerosol deposition, was measured by Constant Temperature Anemometry (CTA).

The StreamLine hot-wire anemometer (Dantec Dynamics A/S, Skovlunde, Denmark) was used for measurement of the velocity in the clean air and aerosol path tubes. Two single-wire probes (Dantec 55P13) were used. The calibration was performed by Dantec StreamLine ${ }^{\circledR}$ Pro Automatic calibrator in the range of 0.02 to $30 \mathrm{~m} / \mathrm{s}$.

The following four cases were measured: 1) the clean air path with disconnected valve (i.e. no closing), 2) the clean air path with connected and activated valve, 3) the aerosol path with disconnected valve, and 4) the aerosol path with connected and activated valve. The measurements were made three times for each case, while nine breath cycles were recorded during each run. 


\section{Results and discussion}

The resulting records of velocity were averaged and interpolated by cubic B spline and compared. The measurements showed that there were no significant differences between the clean air and aerosol path for the cases with disconnected valves. It means that any differences in the courses of velocities in the following cases can be attributed to the role of the valves.

Figs. 9 and 10 show results with connected and activated valves for aerosol and clean air path, respectively. The red curve represents the velocity in the tube from the pneumatic cylinder to the electropneumatic valve, the blue curve represents the velocity in the tube behind the valve branch which is being closed during expiration. The velocities differ before and after the valve, however, it is just a consequence of misalignment of the probes with the centre of the tube and different velocity profiles in the tubes. Both the curves basically overlap during the inspiration for both aerosol and clean air path. However, the delay in the valve closing at the beginning of the expiration can be clearly identified in both graphs. The reaction time is about $0.5 \mathrm{~s}$ and the flow terminates in additional approximately $0.5 \mathrm{~s}$.

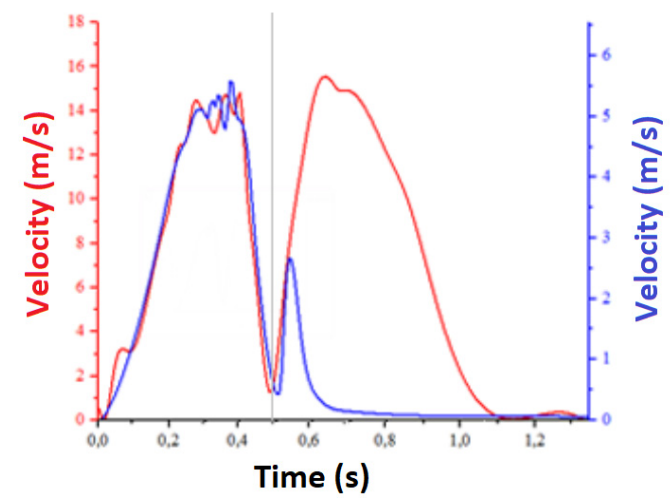

Fig. 9. Velocities before (red) and after (blue) the closing valve on the aerosol path

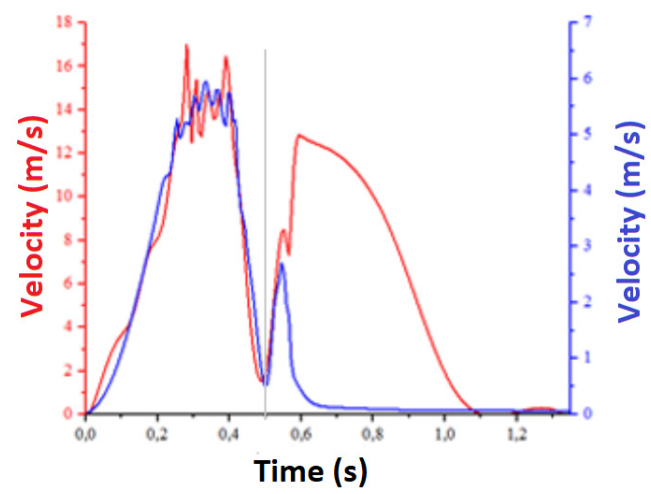

Fig. 10. Velocities before (red) and after (blue) the closing valve on the clean air path

\section{Conclusions}

The results showed that it is necessary to decrease the delay in closing time of the valve. The valves were not originally set to the minimal reaction time, therefore the minimal time of $3.7 \mathrm{~ms}$ was set. The verification of this setup is in progress. Further decrease of the closing delay can be achieved by opening of the valves in advance, however, this setup requires profound changes in the software of the simulator.

The tests also proved that the simulator accurately follows the prescribed breathing pattern and that there were no significant differences in the velocity profile on both aerosol and clean air paths when the electropneumatic valves were open.

Acknowledgement: This work was supported by the Czech Science Foundation under the grant GA16-23675S, by the project FSI-S-17-4444, and by the project LTC17087 of the programme INTER-EXCELLENCE, sub-programme INTERCOST.

\section{References}

1 A. V. Kolanjiyil, C. Kleinstreuer, and R. T. Sadikot, Computers in biology and medicine 84, 247 (2017); B. Hajian, J. De Backer, W. Vos, J. Aerts, J. Cluckers, and W. De Backer, Expert Opinion on Drug Delivery 13 (12), 1719 (2016).

2 Pathway medicine, (2017), Vol. http://www.pathwaymedicine.org/lung-volumes.

3 D. Stejskal, Bachelor thesis, Brno University of Technology, 2017.

4 F. Lizal, J. Jedelsky, K. Morgan, K. Bauer, J. Llop, U. Cossio, S. Kassinos, S. Verbanck, J. Ruiz-Cabello, A. Santos, E. Koch, and C. Schnabel, European Journal of Pharmaceutical Sciences (2017).

5 M. Hussain, W.-H. Renate, and H. Werner, Journal of Thoracic Disease 3 (3), 156 (2011).

6 S. Choi, E. A. Hoffman, S. E. Wenzel, M. H. Tawhai, Y. B. Yin, M. Castro, and C. L. Lin, Journal of Applied Physiology 115 (5), 730 (2013).

7 P. Bokov, B. Mauroy, M. P. Revel, P. A. Brun, C. Peiffer, C. Daniel, M. M. Nay, B. Mahut, and C. Delclaux, Respiratory Physiology \& Neurobiology 173 (1), 1 (2010); C. P. Heussel, S. Ley, A. Biedermann, A. Rist, K. K. Gast, W. G. Schreiber, and H. U. Kauczor, European radiology 14 (12), 2188 (2004).

8 F. Lizal, J. Elcner, P. K. Hopke, J. Jedelsky, and M. Jicha, Proceedings of the Institution of Mechanical Engineers Part H-Journal of Engineering in Medicine 226 (H3), 197 (2012); F. Lizal, M. Belka, J. Adam, J. Jedelsky, and M. Jicha, Proceedings of the Institution of Mechanical Engineers Part H-Journal of Engineering in Medicine 229 (10), 750 (2015).

9 N. Jahani, S. Choi, J. Choi, K. Iyer, E. A. Hoffman, and C. L. Lin, Journal of Applied Physiology 119 (10), 1064 (2015).

10 H. N. E. Bastos, I. Neves, M. Redondo, R. Cunha, J. M. Pereira, A. Magalhaes, and G. Fernandes, J Bras Pneumol 41 (6), 489 (2015).

11 S. G. Yuan, K. A. Frey, M. D. Gross, J. A. Hayman, D. Arenberg, X. W. Cai, N. Ramnath, K. Hassan, J. Moran, A. Eisbruch, R. K. Ten Haken, and F. M. Kong, Int J Radiat Oncol 82 (4), E631 (2012).

12 N. Berend, A. J. Woolcock, and G. E. Marlin, Thorax 35 (2), 145 (1980). 